\title{
Vereinigte Medizinische Gesellschaft in Kiew. Sektion für
} Augenheilkunde

\section{Sitzung vom, 20. März 1930}

Vorsitzender Prof. M. Lewitsky.

1. A. Wassütinsky, , Demonstration eines Falles nach der Ent-fernung von Fibroma cavernosum retrobulbaris ohne Verletzuns desSehnerven".

N., 21 J., bemerkte bei sich vor einem Jahre beginnenden Exophtal-mus des rechten Auges. Vor sechs Monaten wurde in der Augenklinik des Kiewer Instituts für ärztliche Fortbildung rechtseitige Orbitalge-schwulst, die Hervortreten des Auges um $3 \mathrm{~mm}$ verursachte, festgestellt. Punktion o. B. Röntgen und WaR. negativ. Augenhintergrund normal. Visus beiderseitig 0,9. Röntgentherapie resultatlos. Nachfolgend Lagophthalmus und Keratitis. Sehschärfe nahm bis 0,1 ab. Orbito-tomia nach Krönlein. Es gelang, die Geschwulst, die sich gerade im Muskeltrichter befand und den Sehnerv von drei Seiten unigab, ohne Beschädigung des Sehnerves, zu entfernen. Braungraue Geschwulst, die eine Kapsel besaß, erwies sich bei mikroskopischer Untersuchung als Fibroma cavernosum. Nach der Operation Visus $=0,1$, andauernde Ptosis und Beschränkung der Augenbewegungen.

2. A. Rumjanzewa: Fall von Plastik des Unterlides.

Bei Abwesenheit der unteren Übergangsfalte auf dem unteren Lide traumatischen Ursprungs

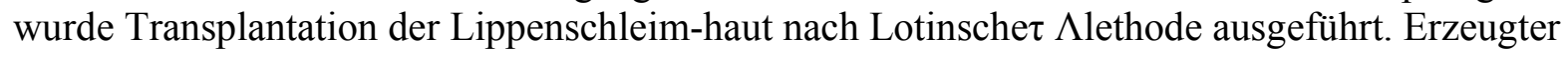
Konjunktivalsack war groß genug für mittelgrol $3 / 8$ e Prothese.

Aussprache: Wassütinsky, Lewitsky, Danilewsky, G. Poljak.

3. Prof. De-Metz (Physiker): Über Radiation kosmischer Strahlen(Übersichtsvortrag).

Sitzung vom 2. April 1930. Vorsitzender Prof. M. Lewitsky.

B. Poljak demonstriert: a) Fall von Lipodermoid Conjunctivae bnlbi,

b) Fall von Limbuscyste (vermutlich traumatischen Ursprunges).

2. M. Lewitsky: Fall von Siderosis bulbi.

Beim Kranken, 48 J., wurde vor acht Monaten das rechte Auge durch einen Eisensplitter verletzt. Der Splitter verblieb in der Linse, wo er sich die ganze Zeit befindet. Das Auge ist vollständig reizlos. Side-rotische traumatische Katarakt. Es wird beabsichtigt, die Linse zusammen mit dem Splitter zu entfernen.

3. W. Kramarewsky: Ultraviolette Strahlen als Hilfsmittel bei derBeliandlun $3 / 8$ der Augenkrankheiten.

Vereinigle Medizinische Gesellschaft in Kiew.

Diskussion.

B. Poljak: Es wird angenommen, daß Frühlingskatarrh durch Ein-wirkung von

Ultraviolettstrahlen entsteht. Darum ist es sehr interessant, daß K. bei der Behandlung so gute

Resultate bekommt.

Danilewsky: Yortr. vergleicht leider die Erfolge verschiedener Methoden nicht, so daß nicht zu ersehen ist, ob seine Behandlungsmethode bessere oder schlechtere Resultate als andere liefert. 
Der Literatur-überblick ist nicht systematisiert und darum die Meinung zahlreicher anderer Autoren nicht erkennbar.

Kiritschinsky: Es ist notwendig, die lokale Behandlung bei ge-schlossenen Lidern anzuwenden. Lewitsky: Diese Methode wird in der Klinik fast ausschließlich bei Skrofulose, Tuberkulose und Trachom angewendet. Die Methode ergibt gute Resultate.

Sitzung vom $\tau 6$. April IÇ30. VorsitzenderProf.A. Wassütinsky.

R. Kruglikowa: Radiumbehandlung eines Kankroids.

Erfolgvolle Behandlung eines Falles von Kankroid, der sich im medialen Winkel des rechten Auges befand und sich schon ziemlich weit in die Orbita erstreckte.

2. Fischmann: Demonstration eines Falles von Fukalascher Operation.Die Resultate befriedigten.

Aussprache: G. Poljak, Samkowsky, Wassütinsky.

B. Poljak: Fall mit eigentümlichen angeborenen Veränderungen auî dem Augenhintergrunde.

R. Elina: Paralysis M·recti superioris eines Auges mit Kontraktur und Strabismus sursum vergens auf dem anderen, unklarer Aetiologie.

5. /. Danilewsky: Erzeugung des künstlichen Konjunktivalsackes nach Duvevge.

6. . A. Rumjanzewa: Operative Belıaklluri! >

des Exophthalmus inter-

mittens (S. diese Zeitschrift Bd. 71

H. 4). G. D. Poljak, 